\title{
Probabilistic Segmentation of the Lumen from Intravascular Ultrasound Radio Frequency Data
}

\author{
E. Gerardo Mendizabal-Ruiz and Ioannis A. Kakadiaris \\ Computational Biomedicine Lab, Departments of Computer Science, \\ Electrical and Computer Engineering, and Biomedical Engineering, \\ University of Houston, Houston, TX
}

\begin{abstract}
Intravascular ultrasound (IVUS) is a catheter-based medical imaging technique that produces cross-sectional images of blood vessels. In this paper, we present a method for the segmentation of the luminal border using IVUS radio frequency (RF) data. Specifically, we parameterize the lumen contour using Fourier series. This contour is deformed by minimizing a cost function that is formulated using a probabilistic approach in which the a priori term is obtained using the prediction confidence of a Support Vector Machine classifier using features extracted from the RF signal. We evaluated the performance of our method by comparing our results with manual segmentations from two expert observers on 280 frames from eight $40 \mathrm{MHz}$ IVUS sequences from rabbits and pigs. The performance was evaluated using the Dice similarity coefficient, coefficient of determination, and linear regressions of the lumen area for each frame. Our results indicate the feasibility of our method for the segmentation of the lumen from IVUS RF data.
\end{abstract}

\section{Introduction}

Intravascular ultrasound (IVUS) is a catheter-based imaging technique that provides high-resolution, cross-sectional images of the interior of blood vessels in real time. The IVUS system consists of a transducer which transmits pulses and receives a reflected radio frequency (RF) signal (i.e., A-line) at a discrete set of angles. These signals are then processed to reconstruct an image that is meaningful to the physicians (i.e., Bmode image). Accurate segmentation of IVUS images is important in order to assess vessel characteristics (e.g., the lumen and wall diameters), and for other applications (e.g., the study of mechanical properties of the vessel wall and the characteristics of the plaque, 3D reconstruction of the vessel). However, manual segmentation of IVUS data can be very expensive considering that one typical sequence may be composed of thousands of frames. Therefore, methods for automatic segmentation of IVUS data are needed.

Early IVUS systems operated at frequencies in the range of 10 to $20 \mathrm{MHz}$. At these frequencies, the blood presents a low acoustic impedance and therefore these systems produce IVUS images in which the lumen has low intensity, no texture, and a high contrast with respect to the vessel wall tissues. For this reason, many approaches for IVUS segmentation were based on the use of local properties of the image such as pixel intensity and gradient information. Modern IVUS systems operate at high frequencies

N. Ayache et al. (Eds.): MICCAI 2012, Part II, LNCS 7511, pp. 454 461, 2012.

(c) Springer-Verlag Berlin Heidelberg 2012 
(i.e., 30 to $40 \mathrm{MHz}$ ) and produce images with high resolution. In these images, the lumen presents some texture due to speckle, and lower contrast with respect to the vessel wall tissues. For these images, edge information is not sufficient and therefore, later approaches incorporated prior knowledge using region and global information such as texture [1], gray level variances [2]3], statistical properties of the intensities [4], temporal information (3D segmentation) [5], and discrete wavelet decomposition [6]. Most recent approaches include the use of nonparametric probability densities with global measurements [7], multilevel discrete wavelet frame decomposition [8], discrete wavelet packet transform [9], machine learning classification methods [10], a combination of gray level probability density functions and the intensity gradient [11], linear-filtered gradient vector flow which drives the deformation of a balloon snake [12], and binary morphological object reconstruction [13].

A common characteristic of these methods is that the segmentation is performed using the reconstructed $\mathrm{B}$-mode images. This poses a limitation considering that, apart from the frequency of operation, the appearance of the B-mode images depends on the reconstruction settings (e.g., time gain compensation (TGC), dynamic range compression, brightness, contrast and scaling) [14] (Figs. 1(a) and 1(b)]. These settings are subjectively selected by the interventionist, and may change from one intervention to the next, or even during the same acquisition [15].

One solution to overcome these limitations is to perform the segmentation employing the raw IVUS RF signal since it is not affected by transformation parameters or visualization settings. RF-based approaches include methods for the characterization of different regions of interest such as plaque or blood (e.g., [16 17]).

In this work, we present a probabilistic method for the segmentation of the lumen from IVUS RF data. Our main contribution is a novel approach for the computation of the a priori terms necessary for a probabilistic segmentation by the analysis of the RF signal instead of the B-mode image. We evaluated the performance of our method by comparing our segmentation results with the manual segmentation from two expert observers on 280 frames from eight $40 \mathrm{MHz}$ IVUS sequences from rabbits and pigs. Our results indicate the feasibility of our method for the segmentation of lumen.

The rest of the paper is organized as follows: Section 2 presents the steps of our segmentation method, Section 3 presents the results obtained with our method, and Sections 4 and 5 present our discussion and conclusion, respectively.

\section{Methods}

The proposed method is a significant improvement of our method presented in [18]. In that work, the segmentation of the lumen was achieved by the minimization of a probabilistic cost function formulated using a Bayesian approach:

$$
U(\boldsymbol{C})=\sum_{r} \sum_{\theta} P_{l}(r, \theta, \boldsymbol{C})\left[-\log \left(v_{l}(r, \theta)\right)\right]+\left[1-P_{l}(r, \theta, \boldsymbol{C})\right]\left[-\log \left(v_{n}(r, \theta)\right)\right],
$$

where $r$ and $\theta$ represent the polar coordinates of each pixel (i.e., radius and angle, respectively), $P_{l}$ is the probability of each pixel to belong to the lumen class, and $v_{l}(r, \theta)$ and $v_{n}(r, \theta)$ represent to the a priori information (i.e., likelihood of the pixel to belong 
to the lumen and non-lumen, respectively). The probability of a pixel to belong to lumen class is defined by its radial distance from the lumen curve $S(\theta, \boldsymbol{C})$ using a sigmoid function: $P_{l}(r, \theta, \boldsymbol{C})=\left(1+\exp ^{-\lambda(S(\theta, \boldsymbol{C})-r)}\right)^{-1}$.

Using this formulation, those pixels far above the contour will have a higher probability of belonging to the lumen, while the pixels far below the contour will have probability close to zero. For the pixels near the contour, depending on the value of $\lambda$, the probability of belonging to lumen will be close to 0.5 .

The two major limitations of our previous method [18] are the number of parameters to be tuned for the parameterization of the curve which uses a mixture of Gaussians, and the simple method for estimating the a priori information models using gray-level histograms which limited the method for the segmentation of $20 \mathrm{MHz}$ IVUS sequences exclusively. The proposed method has two significant differences with respect to that work: (i) we propose a parameterization which requires less parameters to be tuned and that suits better to the characteristics of the segmentation problem, and (ii) the computation of the a priori terms is based on the analysis of the RF signal.

The RF IVUS data corresponding to a cross section of a vessel consist of a set of one-dimensional signals. Each of these signals corresponds to the acoustic echoes acquired at a particular angle using a single-rotating transducer or a circular array of transducers. Therefore, the data can be analyzed using polar coordinates where the radius corresponds to the penetration of the ultrasound beam measured from the transducer (Fig. [1(d)]. In this context, the lumen contour can be represented as a one-dimensional periodic curve. In this work, we have chosen to define the lumen curve using onedimensional Fourier series instead of other parameterizations due to its simplicity and because this parameterization provides a periodic curve for which the smoothness can be controlled by the number of harmonics (i.e., number of coefficients $N_{k}$ ). The lumen contour is defined by:

$$
S(\theta, \boldsymbol{C})=\frac{a_{0}}{2}+\sum_{k=1}^{N_{k}}\left[a_{k} \cos \left(\frac{k 2 \pi \theta}{N_{\theta}}\right)+b_{k} \sin \left(\frac{k 2 \pi \theta}{N_{\theta}}\right)\right],
$$

where $N_{\theta}$ represents to the number of A-lines in a frame (i.e., width of the polar Bmode image), and $\boldsymbol{C}=\left[a_{0}, a_{1}, \ldots, a_{N_{k}-1}, b_{1}, \ldots, b_{N_{k}-1}\right]^{T}$ are the Fourier coefficients that control the shape of the curve.

For the computation of the a priori terms, we employ features extracted from the IVUS RF signal using our blood detection method proposed in [17]. In that work, the structures present in the vessel are modeled as a distribution of random positioned scatterers for which the acoustic power scattered in the direction of the ultrasound transducer is defined by the differential backscattering cross section (DBC) of the scatterer. The RF signal corresponding to each A-line is divided into $N_{P}$ non overlapping partitions of constant width. The DBC of the scatterers that generates the signal of each partition is computed by minimizing the difference between the root mean square power of the real RF signal and the modeled RF signal computed with the model. In this work, we employ the DBC and the radial distance of the partition as features to detect the blood and non-blood regions using a machine learning method for classification.

In summary, our method consists of six steps: (i) divide the RF signal corresponding to each A-line of a frame into $N_{P}$ non-overlapping partitions; (ii) compute the DBC 
values for all the partitions using the method described in [17]; (iii) construct a feature vector $v_{p} \in R^{2}$ for each partition $p$ by concatenating the DBC computed for that partition and its radial distance from the transducer; (iv) classify each partition as blood or non-blood according to the prediction of a classifier; (v) use the confidence of the classification results as the a-priori term in the Bayesian optimization function; and (vi) minimize the cost function that guides the position and shape of the lumen contour using a line-search method.

In this work, we chose to use a Support Vector Machines (SVM) classifier in order to take advantage of its ability of mapping the data to a higher dimensional space on which the problem becomes linearly separable. The training and deployment steps for the SVM model are described next.

Training: In the training step, the user to provides examples of lumen and non-lumen regions by manual annotations of these regions on the first frame B-mode reconstruction of the sequence to be segmented (Figs. 1(b) and 1(c)]. Next, the DBC of the scatterers that generate the signal of each partition corresponding to the regions provided as examples is computed. A two-dimensional feature vector $v_{p} \in R^{2}$ is constructed for each partition. A training set is defined by associating each vector with its corresponding class and then used to generate an SVM model that is trained using a Gaussian kernel. The optimal parameters $c$ and $\gamma$ for the SVM model are computed using grid search and 5 -fold cross validation.

Deployment: For each of the frames to be segmented, the DBC of the scatterers for each partition of every A-line is computed, and the feature vector is constructed in the same way as in the training step. The classification of each partition is performed using the SVM model giving as a result a class and an associated confidence $P_{s}$ (Fig. 1(e)]. This classification confidence is then used as the likelihood for blood and non-blood as $v_{l}(r, \theta)=P_{s}(r, \theta)$ and $v_{n}(r, \theta)=1-P_{s}(r, \theta)$, respectively. Finally, the segmentation is performed by the minimization of the cost function of Eq. 11 using the steepest descent method.

\section{Results}

We use the proposed method to perform segmentation on selected frames from eight $40 \mathrm{MHz}$ IVUS sequences, four were acquired from rabbits and the other four from pigs. For each sequence, we selected five groups of seven consecutive frames from different periods of time for a total of 35 frames per sequence. The parameters used for the computation of the DBC are $\sigma=5.3 e^{-8}$ for the width of the envelope of the impulse function, $\mu=0.08276 \mathrm{~dB} / \mathrm{mm}$ corresponding to the attenuation coefficient of blood, $c=1,540 \times 10^{3} \mathrm{~mm} / \mathrm{s}$ corresponding to the speed of sound in biological tissue, $\triangle P=0.05 \mathrm{~mm}$ for the size of partition, $D=400$ scatterers $/ \mathrm{mm}^{-2}$ for the scatterer density, and $\delta=3$ for the cardinality of neighbors according to [17]. The number of coefficients used for the contour parameterization was $N_{k}=5$. The parameter for the probabilistic segmentation was experimentally set to $\lambda=0.4$. The initial point for the 


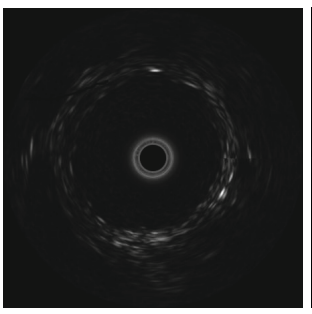

(a)

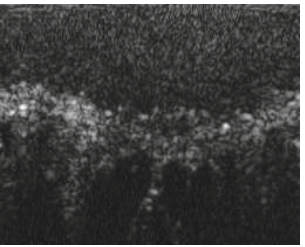

(d)

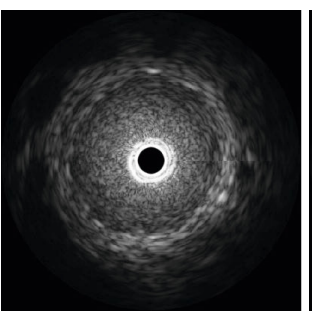

(b)

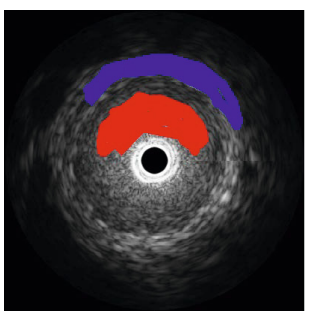

(c)

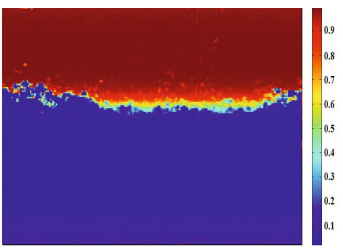

(e)

Fig. 1. Example depicting (a) the first frame of a sequence in Cartesian B-mode representation using logarithmic dynamic range compression, (b) the same frame using linear dynamic range compression with a compression factor of 0.4 , (c) the corresponding user annotation for blood and non-blood (red and blue, respectively), (d) the polar B-mode representation of the frame, and (e) the probability of each partition to belong to blood according to the SVM model in polar representation (color figure).

steepest descent optimization was empirically set to $a_{0}=1, a_{i}=0.1$ and $b_{i}=0.1$ $\forall i>0$. The method was implemented in MATLAB and the average time for training the SVM for each sequence was $12 s$ and the segmentation time per frame was $5.4 s$ on an Intel i7 at $2.67 \mathrm{GHz} 12 \mathrm{~GB}$ RAM.

We evaluated the performance of our method by comparing our segmentation results (A) with the manual segmentation from two expert observers $\left(\mathrm{O}_{1}\right.$ and $\left.\mathrm{O}_{2}\right)$. We computed the Dice similarity coefficient for the comparison of segmentations of $\left(\mathrm{O}_{1}\right.$ vs. $\left.\mathrm{O}_{2}\right),\left(\mathrm{O}_{1}\right.$ vs. A), and $\left(\mathrm{O}_{2}\right.$ vs. A) for each frame. Figure 2 depicts the results of this analysis for each sequence and for the total number of frames. Note that our segmentation results are similar to the segmentation provided by the two expert observers. In addition, we evaluated the agreement between the areas defined by each segmentation by computing the coefficient of determination and using linear regressions (Fig. 3). Although there exists a good agreement between the areas, a tendency of our method to under-segment the areas can be observed. Finally, Figure 4 depicts examples of the segmentation results along with the manual segmentations from the two expert observers. 


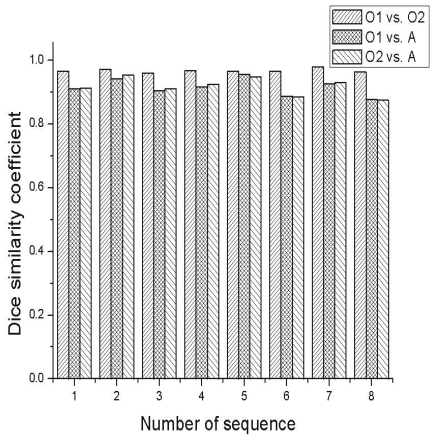

(a)

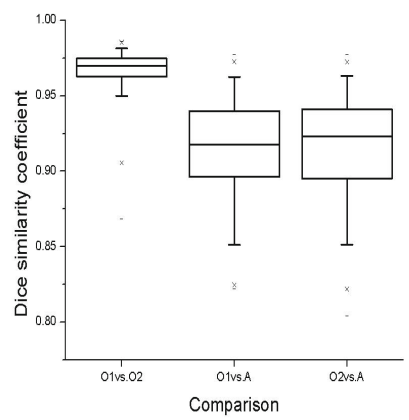

(b)

Fig. 2. Mean Dice similarity coefficient for (a) each sequence, and (b) the total number of frames

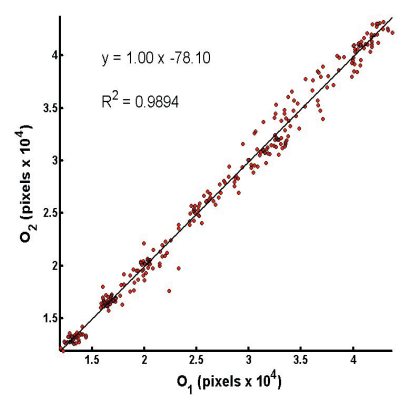

(a)

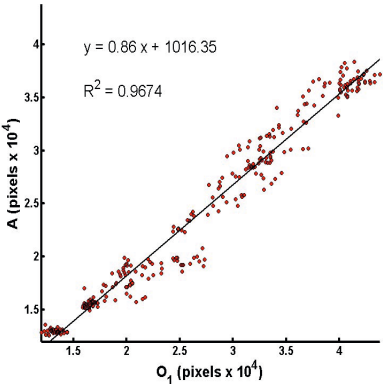

(b)

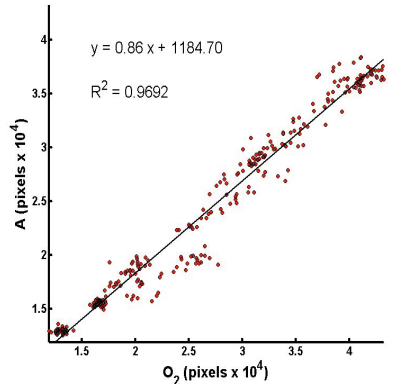

(c)

Fig. 3. Linear regression and coefficient of determination for the comparisons of (a) $\left(\mathrm{O}_{1}\right.$ vs. $\left.\mathrm{O}_{2}\right)$, (b) $\left(\mathrm{O}_{1}\right.$ vs. A), and (c) $\left(\mathrm{O}_{2}\right.$ vs. A)
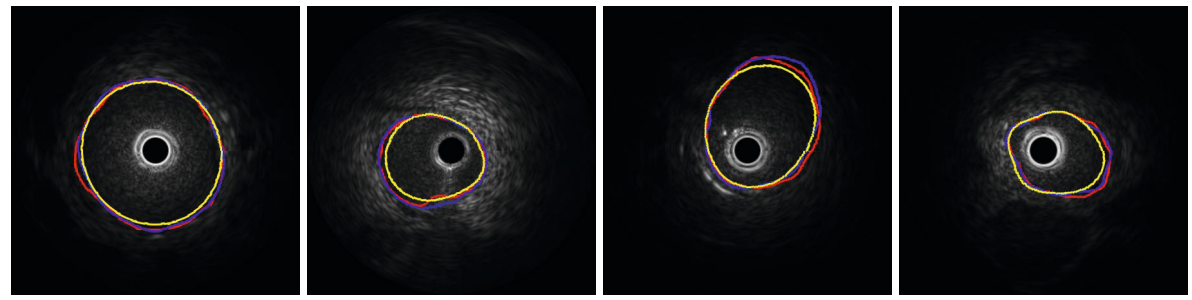

Fig. 4. Examples of segmentation results (yellow) and their qualitative comparison with manual segmentations 


\section{Discussion}

The main advantage of our method when compared with other existing IVUS segmentation methods is that our method is not affected by the B-mode reconstruction parameters since it is based on the use of the RF signal. While there may be a concern regarding the availability of such data, note that recent IVUS systems available in the clinic are capable of providing the RF signal. The selected dataset included frames with moderate guidewire artifacts and did not include frames with strong guidewire artifacts, plaque, stents and side branches as this is subject for future work. In this work, we do not evaluate the accuracy of the classification method in the classification of a frame since this data is not used as a direct result of the segmentation method but instead as a likelihood of a pixel to belong to a certain class. The final class assigned to each pixel is determined by the segmentation curve. Real-time segmentation may be achieved by an implementation in a lower level language and by segmenting frames in parallel. Additionally, the proposed method may be fully automated by creating an SVM model using samples from a large dataset of sequences instead of training for each sequence. The main limitation of our method is the requirement of user initialization. Additionally, our method may provide an incorrect segmentation in the presence of side branches for which expert knowledge is required. Improvements on the classification performance, the use of other classification methods, different parameterizations of the lumen curve, exploration of additional features, including additional classes apart from blood and non-blood (e.g., guidewire, shadow, plaque.), segmentation of the media/adventitia interface, the use of temporal information (i.e., 3D-approach), and comparison with other existing methods are subjects of future research.

\section{Conclusion}

We have presented a novel approach for the segmentation of the luminal border using IVUS RF data. Our results indicate the feasibility of our method for the segmentation of lumen in real time applications.

\section{References}

1. Mojsilovic, A., Popovic, M., Amodaj, N., Babic, R., Ostojic, M.: Automatic segmentation of intravascular ultrasound images: A texture-based approach. Annals of Biomedical Engineering 25(6), 1059-1071 (1997)

2. Haas, C., Ermert, H., Holt, S., Grewe, P., Machraoui, A., Barmeyer, J.: Segmentation of 3D intravascular ultrasonic images based on a random field model. Ultrasound in Medicine and Biology 26(2), 297-306 (2000)

3. Luo, Z., Wang, Y., Wang, W.: Estimating coronary artery lumen area with optimization-based contour detection. IEEE Transactions on Medical Imaging 22, 564-566 (2003)

4. Brusseau, E., de Korte, C.: Fully automatic luminal contour segmentation in intracoronary ultrasound imaging - a statistical approach. IEEE Transactions on Medical Imaging 23(5), 554-566 (2004)

5. Cardinal, M., Meunier, J., Soulez, G., Maurice, R., Therasse, E., Cloutier, G.: Intravascular ultrasound image segmentation: A three-dimensional fast-marching method based on gray level distributions. IEEE Transactions on Medical Imaging 25(5), 590-601 (2006) 
6. dos Santos Filho, E., Yoshizawa, M., Tanaka, A., Saijo, Y.: A study on intravascular ultrasound image processing. Record of Electrical and Communication Engineering Conversazione 74(2), 30-33 (2006)

7. Unal, G., Bucher, S., Carlier, S., Slabaugh, G., Fang, T., Tanaka, K.: Shape-driven segmentation of the arterial wall in intravascular ultrasound images. IEEE Transactions on Information Technology in Biomedicine 12(3), 335-347 (2008)

8. Papadogiorgaki, M., Mezaris, V., Chatzizisis, Y., Giannoglou, G., Kompatsiaris, I.: Image analysis techniques for automated IVUS contour detection. Ultrasound in Medicine and Biology 34(9), 1482-1498 (2008)

9. Katouzian, A., Baseri, B., Konofagou, E., Laine, A.: Automatic detection of blood versus non-blood regions on intravascular ultrasound (IVUS) images using wavelet packet signatures. In: Proc. SPIE Medical Imaging 2008: Ultrasonic Imaging and Signal Processing, San Diego, CA, February 16-21 (2008)

10. Ciompi, F., Pujol, O., Fernández-Nofrerías, E., Mauri, J., Radeva, P.: ECOC Random Fields for Lumen Segmentation in Radial Artery IVUS Sequences. In: Yang, G.-Z., Hawkes, D., Rueckert, D., Noble, A., Taylor, C. (eds.) MICCAI 2009, Part II. LNCS, vol. 5762, pp. 869876. Springer, Heidelberg (2009)

11. Cardinal, M., Soulez, G., Tardif, J., Meunier, J., Cloutier, G.: Fast-marching segmentation of three-dimensional intravascular ultrasound images: A pre-and post-intervention study. Medical Physics 37(7), 3633-3647 (2010)

12. Zhu, X., Zhang, P., Shao, J., Cheng, Y., Zhang, Y., Bai, J.: A snake-based method for segmentation of intravascular ultrasound images and its in vivo validation. Ultrasonics 51(2), 181-189 (2011)

13. Moraes, M., Furuie, S.: Automatic coronary wall segmentation in intravascular ultrasound images using binary morphological reconstruction. Ultrasound in Medicine and Biology 37, 1486-1499 (2011)

14. Hiro, T., Leung, C., Russo, R., Karimi, H., Farvid, A., Tobis, J.: Variability of a three-layered appearance in intravascular ultrasound coronary images: A comparison of morphometric measurements with four intravascular ultrasound systems. American Journal of Cardiac Imaging 10(4), 219-227 (1996)

15. Mintz, G., Nissen, S., Anderson, W., Bailey, S., Erbel, R., Fitzgerald, P., Pinto, F., Rosenfield, K., Siegel, R., Tuzcu, E., Yock, P.: American College of Cardiology clinical expert consensus document on standards for acquisition, measurement and reporting of intravascular ultrasound studies (IVUS). Journal of the American College of Cardiology 37(5), 1478-1492 (2001)

16. Nair, A., Margolis, M., Kuban, B., Vince, D.: Automated coronary plaque characterisation with intravascular ultrasound backscatter: Ex vivo validation. Eurointervention 3(1), $113-$ 120 (2007)

17. Mendizabal-Ruiz, E., Biros, G., Kakadiaris, I.A.: Towards Extra-Luminal Blood Detection from Intravascular Ultrasound Radio Frequency Data. In: Fichtinger, G., Martel, A., Peters, T. (eds.) MICCAI 2011, Part I. LNCS, vol. 6891, pp. 396-403. Springer, Heidelberg (2011)

18. Mendizabal-Ruiz, E., Rivera, M., Kakadiaris, I.: A probabilistic segmentation method for the identification of luminal borders in intravascular ultrasound images. In: Proc. IEEE Computer Society Conference on Computer Vision and Pattern Recognition, Anchorage, AK, June 2426, pp. 1-8 (2008) 